\title{
Traqueostomía percutánea en cuidado intensivo: una opción rápida, fácil y segura
}

\author{
Percutaneus tracheostomy in intensive care, a quick, \\ easy and safe option
}

Carlos Laverde-Sabogal ${ }^{1}$, Oscar Moreno-Ojeda², Daniela Patiño-Hernández ${ }^{3}$

\begin{abstract}
Invasive mechanical ventilation (IMV) is one of the most frequent causes of admission to the Intensive Care Unit (ICU), when such support is prolonged and its removal is not possible, tracheostomy emerges as the only alternative. We seek to identify and describe the clinical outcomes of patients in IMV undergoing a percutaneous tracheostomy technique. We present a descriptive cross-sectional study based on a cohort of adult patients with IMV admitted to the ICU between January 1, 2017 and June 30, 2019, performing the percutaneous tracheostomy technique at the patient's bedside. Demographic, intervention and outcome variables were collected. We found 46 patients, with an average age of 52.6 years (DE), $69.57 \%$ corresponded to male sex. The most frequent indication was the presence of neurological disorders, and the average number of days until its completion was $10.36 \%$. 36.96\% were performed by the intensivists and the remaining $63.04 \%$ by general surgeons, their percentage of complications was diferent, compared to $7.4 \%$ worldwide. The most frequent complication was the pneumothorax $6.5 \%$. We did not have mortality or infections. Percutaneous tracheostomy is a safe, fast and efficient alternative achievable in the patient's bedside by the trained anesthesiologist.
\end{abstract}

\section{RESUMEN}

La ventilación mecánica invasiva (VMI) es una de las causas más frecuentes de ingreso en la Unidad de Cuidado Intensivo (UCI), cuando dicho soporte se

\section{Key words:}

Percutaneous

tracheostomy, mechanical ventilation release, complications

\section{Palabras clave:}

Traqueostomía

percútanea, liberación mecánica, complicaciones

\footnotetext{
Anestesiólogo Intensivista Unidad de Cuidado Intensivo, Hospital Universitario San Ignacio. Profesor Asistente Pontificia Universidad Javeriana.

2 Médico Intensivista Unidad de Cuidado Intensivo Hospital Universitario San Ignacio.

3 Residente de Medicina Interna. Departamento de Medicina Interna. Hospital Universitario San Ignacio y Pontificia Universidad Javeriana.
}

Fecha de recepción: 19 de diciembre de 2019

Fecha de aceptación: 02 de enero de 2020

\section{ORCID}

http://orcid.org/0000-0001-6017-3059

Correspondencia:

Carlos Laverde-Sabogal

kakayy@gmail.com 
prolonga y no es posible su retiro surge la traqueostomía como única alternativa. Buscamos identificar y describir los desenlaces clínicos de pacientes en VMI sometidos a una técnica de traqueostomía percutánea. Presentamos un estudio descriptivo de corte transversal basado en una cohorte de pacientes adultos con VMI ingresados en la UCl entre el 1 de enero de 2017 y 30 de junio de 2019, realizándoles la técnica percutánea de traqueostomía en la cabecera del paciente. Se recolectaron variables demográficas, de intervención y desenlace. Encontramos 46 pacientes, con una media de edad de 52,6 años (DE), el $69,57 \%$ correspondía a sexo masculino. La indicación más frecuente fue la presencia de trastornos neurológicos, y la media de días hasta su realización fue de 10,36. El 36,96\% fueron realizadas por los intensivistas y el 63,04\% restante por cirujanos generales, su porcentaje de complicaciones fue diferente, frente a $7,4 \%$ a nivel mundial. La complicación más frecuente fue el neumotórax $6,5 \%$. No tuvimos mortalidad ni infecciones. La traqueostomía percutánea es una alternativa segura, rápida y eficiente realizable en la cabecera del paciente por el anestesiólogo entrenado.

\section{Introducción}

- I soporte ventilatorio invasivo es una de las cau- sas más frecuentes de ingreso de nuestros pacientes al cuidado intensivo, su liberación no es posible en escenarios caracterizados por desacondicionamiento físico, trastornos de deglución o por su patología de base. En este contexto, la traqueostomía se constituye como una alternativa terapéutica con el fin de evitar las complicaciones asociadas a la ventilación prolongada, siendo una opción entre el 8 al 24\% de los pacientes[1]-[3].

Existen dos técnicas descritas para la realización de la traqueotomía, abierta y percutánea, esta última descrita en 1985 por Ciaglia. Los beneficios fisiológicos son la disminución del trabajo respiratorio, el atrapamiento aéreo, la presión inspiratoria, la resistencia de la vía aérea y el espacio muerto. Facilitando así el retiro ventilatorio, la sincronía ventilatoria y los cuidados de enfermería y fisioterapia[2], mejorando el confort y bienestar del paciente[1],[3]. Con la técnica percutánea, disminuyen el tiempo de realización, el recurso humano, la estancia en cuidado intensivo y no se necesita un quirófano por realizarse en la cabecera del paciente[4],[5]. Las dos técnicas descritas son seguras[1]-[3].

Nuestro trabajo busca identificar y describir los desenlaces clínicos de pacientes con requerimiento ventilatorio prolongado sometidos a traqueostomía percutánea en cuidado intensivo.

\section{Materiales y Métodos}

Nuestro estudio es descriptivo de corte transver- sal basado en una cohorte de pacientes mayores de 16 años con soporte ventilatorio invasivo ingresados a la $\mathrm{UCl}$ entre el 1 de enero de 2017 y 30 de junio de 2019 de nuestro hospital, realizándoles la técnica percutánea de traqueostomía de dilatación única. Se recolectaron variables demográficas, de intervención y desenlace. Se describe la muestra mediante: medias, desviaciones estándar, número global y porcentaje según la naturaleza de las variables. Dentro de los criterios de inclusión se tuvieron en cuenta la edad descrita y el requerimiento de ventilación mecánica mayor a 7 días (prolongada). La definición de ventilación prolongada no ha podido ser construida claramente, para efectos operativos de este trabajo manejaremos mayor de 7 días, igualmente no hay un consenso acerca de aquello que se considera una traqueostomía temprana o tardía[5]-[7]. Los criterios de exclusión fueron los pacientes trasladados, con inestabilidad cervical no corregida o coagulopatía activa.

Esta investigación sigue las leyes internacionales para la investigación biomédica de la Organización Mundial de la Salud (OMS) encomendada en la Declaración de Helsinki, y está regulada por la Resolución No 8430 de 1993 del Ministerio de Salud de Colombia. Se considera el presente estudio una investigación de riesgo mínimo al tratarse de recolección retrospectiva de datos, por lo que el Comité de Ética decidió eximir el consentimiento informado.

La técnica de traqueostomía percutánea realizada en este trabajo es de dilatación única, y se llevó a cabo con un kit TRACOE Twist ${ }^{\circledR}$ de cánula no fenestrada con balón[8]-[13]. No se utiliza ecografía ni fibrobroncoscopia. La sedación se realizó con fentanil (1 mcg/kg) y propofol $(1,5 \mathrm{mg} / \mathrm{kg})[2],[8],[9]$. Sin re- 
lajación muscular. Los parámetros del ventilatorios son: modo A/C con $\mathrm{FiO}_{2} 100 \%$ [8],[9]. En relación a la anestesia, se utiliza lidocaína simple al $1 \%$ sin epinefrina para infiltrar piel, tejido celular subcutáneo del sitio seleccionado que corresponde al espacio entre el 2 y 3 anillo traqueal, sumado al bloqueo del nervio laríngeo recurrente bilateral ubicación por reparos anatómicos[2],[8],[9]. La confirmación de la cánula de traqueostomía se realiza combinando la auscultación torácica de 5 puntos, la curva adecuada de capnografía y las presiones obtenidas por el ventilador mecánico de la UCI[8]. Después de la realización de traqueostomía, se realizó radiografía de tórax postprocedimiento para detección de complicaciones en todos los pacientes.

\section{Resultados}

Obtuvimos 46 pacientes con una media de edad de 53,2 años (DE), en su mayoría hombres (69,57\%), mayores de 60 años (43,5\%). Su peso medio de 70,9 $\mathrm{kg}$ en un $16,6 \%$ de la cohorte (Tabla 1 ).

Sus diagnósticos de ingreso más frecuente fue las patologías del sistema nervioso con $54,4 \%$, seguidas por el sistema respiratorio con $19,6 \%$ e infecciosas con 10,9\% (Tabla 1).

Los criterios de ingreso a UCl fueron el soporte ventilatorio $(47,8 \%)$, la vigilancia postoperatoria $(17,4 \%)$ y el soporte vasoactivo sumado al ventilatorio $(10,9 \%)$ (Tabla 1). El puntaje APACHE IV medio fue de 36,8 puntos para $20,2 \%$ de la cohorte que permanecieron más de $48 \mathrm{~h}$ en cuidado intensivo. En cuanto a la estancia media en cuidado intensivo fue de 24,6 días y hospitalaria de 43,7 días (Tabla 1). La estancia en $\mathrm{UCl}$ promedio hasta la realización de traqueostomía fue de 10,36 días. En nuestro estudio se consideró entre las variables de interés, los días hasta retiro del ventilador a manera de variable continua y los datos de días hasta retiro completo de ventilación mecánica se encuentran en la Tabla 2 (representados mediante media y desviación estándar o mediana y rango intercuartílico según distribución de las variables). Se estudió adicionalmente como variable categórica, con la misma agrupación considerada en el estudio WIND[14] con los siguientes resultados: tiempo con ventilación $<1$ día: $\mathrm{n}=2(4,35 \%), 1-7$ días $\mathrm{n}=$ $2(4,35 \%)$, > 7 días $n=42(91,39)$. Todos los pacientes fueron liberados del soporte ventilatorio en modo CPAP y disminución de su presión de soporte (PS) intercalando progresivamente con tiempo en tienda de traqueostomía. Las indicaciones de traqueostomía más frecuentes fueron los trastornos neurológicos
$(45,7 \%)$, la ventilación prolongada (36,9\%), los trastornos de deglución $(15,2 \%)$ y un único paciente por desacondicionamiento físico en la Tabla 2. No existieron casos de mortalidad asociada a traqueostomía, los diagnósticos de ingreso asociados a los casos de mortalidad no asociada a traqueostomía se encuentran en la Tabla 1.

La realización de los procedimientos estuvo a cargo de dos grupos: el primer grupo conformado por intensivistas (doble titulación) con 36,96\% de los procedimientos y un segundo grupo de cirujanos generales realizó el 63,04\% restante. Las complicaciones tuvieron la siguiente distribución: 4,3\% y 21,73\% respectivamente. La complicación más frecuente fue el neumotórax $6,5 \%$ (Tabla 3). No se presentaron complicaciones tempranas (menores de 7 días) que están relacionadas con la obstrucción y el desalojo de la cánula, ni tampoco tardías (mayores de 7 días) que incluyen fístulas, estenosis traqueal y traqueomalacia[1],[8]. No se presentaron infecciones ni mortalidad.

Encontramos una disminución en el tiempo de respuesta de la realización de la traqueostomía en los dos intervalos de tiempo examinados, 2014 - 2016 y 2017 - 2019, de 4,28 días a 2,11 días respectivamente. Este parámetro institucional es evaluado desde la generación de la interconsulta hasta la realización del procedimiento y debe ser de 2 días (Tabla 2).

\section{Discusión}

Los hallazgos más importantes de nuestro estudio fue describir una población latinoamericana con unas características sociodemográficas e indicaciones de traqueostomía percutánea similares a los reportados a nivel mundial. Con la técnica percutánea, mejoramos el tiempo de respuesta para su realización frente al intervalo de tiempo inmediatamente anterior acercándonos al parámetro institucional. Las complicaciones perioperatorias se distribuyeron de acuerdo al grupo que intervino, estas incluyeron pérdida de la vía aérea, inserción paratraqueal, laceración esofágica o traqueal, hemorragia, neumotórax y enfisema subcutáneo[3],[8]. El grupo de cirugía general presentó un porcentaje mayor al parámetro internacional situado en $7,4 \%$, posiblemente por tener personal en elaboración de su curva de aprendizaje. El neumotórax fue nuestra principal complicación. No encontramos diferencias estadísticamente significativas con respecto a complicaciones entre pacientes en quienes se realizó traqueostomía percutánea por intensivistas vs cirujanos generales (Tabla 3). No hubo ningún caso de 


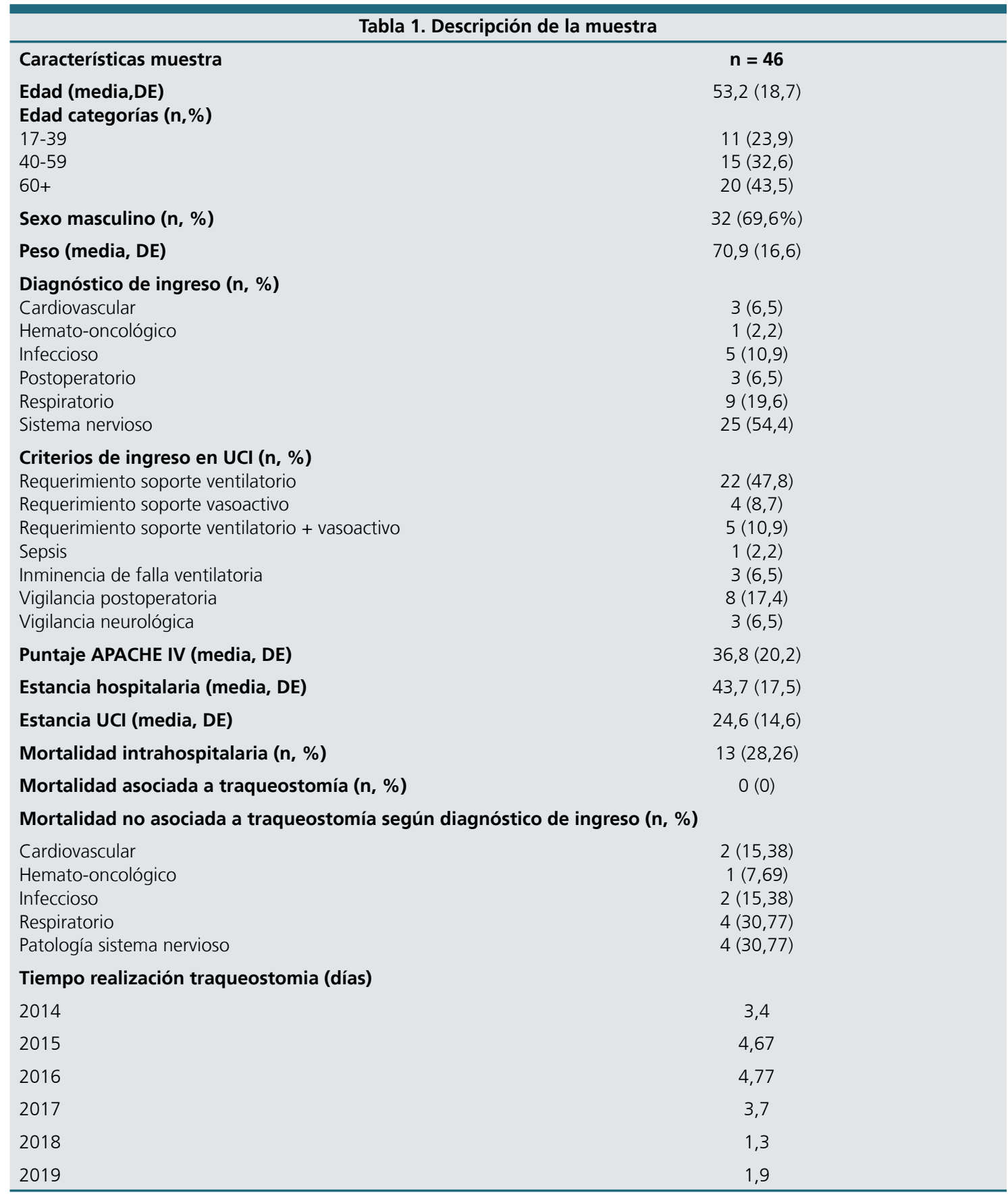

mortalidad asociado a la realización de traqueostomía percutánea durante el periodo de seguimiento en la Unidad de Cuidado Intensivo ni en el resto del seguimiento intrahospitalario En cuanto a la mortalidad a 28 días, al no realizarse seguimiento posterior al egreso, no fue posible evaluar dicha variable dentro de nuestro estudio. No se requirió cambio de técnica quirúrgica percutánea abierta por imposibilidad de la realización del procedimiento. En general la técnica percutánea presenta menores complicaciones frente 


\section{Tabla 2. Variables asociadas a traqueostomía}

\begin{tabular}{lc}
\hline Características muestra & $\mathbf{n = 4 6}$ \\
Indicación de traqueostomía (n, \%) & $17(36,9)$ \\
Ventilación prolongada & $21(45,7)$ \\
Trastorno neurológico & $7(15,2)$ \\
Trastorno de la deglución & $1(2,2)$ \\
Desacondicionamiento físico & $10,4(4,8)$ \\
Días de ventilación mecánica previo a traqueostomía (media,DE) & $2(1-11)$ \\
Días de ventilación mecánica desde traqueostomía hasta egreso (mediana,rango p25-75) & $17(37)$ \\
Especialidad que lleva a cabo procedimiento (n,\%) & $29(63)$ \\
Cuidado Intensivo & $\mathbf{1 2}(\mathbf{2 6 , 1 )}$ \\
Cirugía General & $3(6,5)$ \\
Complicaciones (n, \%) & $2(4,4)$ \\
Neumotórax & $0(0)$ \\
Lesión vascular & $1(2,2)$ \\
Infección sitio operatorio & $5(10,9)$ \\
Hemorragia & $1(2,2)$ \\
Falsa ruta & $0(0)$ \\
Enfisema subcutáneo & $0(0)$ \\
Laceración &
\end{tabular}

\begin{tabular}{lccc} 
& \multicolumn{3}{c}{ Tabla 3. Complicaciones según operador } \\
\hline Complicaciones $(\mathbf{n}, \boldsymbol{\%})$ & $\mathbf{C x}$ & $\mathbf{U C l}^{*}$ & $\mathbf{p}$ valor \\
Neumotórax & $2(6,90)$ & $1(5,88)$ & 0,893 \\
Lesión vascular & $2(6,90)$ & $0(0)$ & 0,268 \\
Hemorragia & $1(3,45)$ & $0(0)$ & 0,439 \\
Falsa ruta & $4(13,79)$ & $1(5,88)$ & 0,405 \\
Enfisema subcutáneo & $1(3,45)$ & $0(0)$ & 0,439 \\
\hline
\end{tabular}

*UCl: Especialistas en Cuidado Intensivo; ${ }^{*}$ Cx: Especialistas en Cirugía General.

a la abierta[2], con menor prevalencia de infección y sangrado[1]-[3],[6].

Dentro de las limitaciones del estudio encontramos el tamaño de la muestra para poder evaluar complicaciones de acuerdo al grupo a cargo de su realización, infecciones y mortalidad. A nivel mundial están en $7,4 \%, 5,9 \%$ y en $0,3 \%$ respectivamente[6],[7].

Para disminuir el riesgo de complicaciones la utilización de fibrobroncoscopia y ecografía no encuentran diferencia. En cuanto a la curva de aprendizaje por reparos anatómicos, las recomendaciones están entre 5 a 20 procedimientos[6]-[8],[13]. La utilización de ecografía presenta una curva más larga para al- canzar un tiempo promedio de procedimiento de 22 minutos y menores complicaciones se requieren al menos 50 procedimientos[15].

\section{Conclusiones}

La traqueostomía percutánea es una alternativa segura, rápida y eficiente realizable en la cabecera del paciente por un anestesiólogo entrenado, al disminuir el tiempo de espera para su realización y así facilitando el retiro ventilatorio, de esta manera mejoramos el giro-cama en Cuidado Intensivo e igualmente se optimizan los recursos de salas de cirugía. 


\section{Referencias}

1. Klotz $R$, Probst $P$, Deininger $M$, Klaiber U, Grummich K, Diener $M K$, et al. Percutaneous versus surgical strategy for tracheostomy: a systematic review and meta-analysis of perioperative and postoperative complications. Langenbecks Arch Surg. 2018;403(2):137-49. https://doi. org/10.1007/s00423-017-1648-8

2. Kidane B, Pierre AF. From Open to Bedside Percutaneous Tracheostomy. Thorac Surg Clin. 2018;28(3):263-76. https://doi.org/10.1016/j.thorsurg.2018.03.001

3. Johnson-Obaseki S, Veljkovic A, Javidnia H. Complication rates of open surgical versus percutaneous tracheostomy in critically ill patients. Laryngoscope. 2016;126(11):2459-67. https:// doi.org/10.1002/lary.26019

4. deBoisblanc BP. Percutaneous dilational tracheostomy techniques. Clin Chest Med. 2003;24(3):399-407. https:// doi.org/10.1016/S02725231(03)00043-1

5. Bittner EA, Schmidt UH. The ventilator liberation process: update on technique, timing, and termination of tracheostomy. Respir
Care. 2012;57(10):1626-34. https://doi.org/10.4187/respcare.01914

6. Raimondi N, Vial MR, Calleja J, Quintero A, Cortes A, Celis E, et al. Evidence-based guidelines for the use of tracheostomy in critically ill patients. J Crit Care. 2017;38:304-18. https://doi. org/10.1016/j.jcrc.2016.10.009

7. Cheung NH, Napolitano LM. Tracheostomy: epidemiology, indications, timing, technique, and outcomes. Respir Care. 2014;59(6):895-915; discussion 6-9. https://doi.org/10.4187/ respcare.02971

8. Mehta C, Mehta Y. Percutaneous tracheostomy. Ann Card Anaesth. 2017;20(Supplement):S19-s25. https://doi.org/10.4103/09719784.197793

9. Rogers S, Puyana JC. Bedside percutaneous tracheostomy in the critically ill patient. Int Anesthesiol Clin. 2000;38(3):95-110. https:// doi.org/10.1097/00004311200007000-00007

10. Cools-Lartigue J, Aboalsaud A, Gill H, Ferri L. Evolution of percutaneous dilatational tracheostomy--a review of current techniques and their pitfalls. World
J Surg. 2013;37(7):1633-46. https://doi.org/10.1007/s00268013-2025-6

11. Durbin CG, Jr. Techniques for performing tracheostomy. Respir Care. 2005;50(4):488-96.

12. Susarla SM, Peacock ZS, Alam HB. Percutaneous dilatational tracheostomy: review of technique and evidence for its use. J Oral Maxillofac Surg. 2012;70(1):74-82. https://doi. org/10.1016/j.joms.2011.04.002

13. Hsia DW, Ghori UK, Musani Al. Percutaneous dilational tracheostomy. Clin Chest Med. 2013;34(3):515-26. https://doi. org/10.1016/j.ccm.2013.04.002

14. Béduneau G, Pham T, Schortgen $F$, Piquilloud L, Zogheib E, Jonas $M$, et al. Epidemiology of Weaning Outcome according to a New Definition. The WIND Study. Am J Respir Crit Care Med. 2017;195(6):772-83. https:// doi.org/10.1164/rccm.20160203200C

15. Petiot $S$, Guinot PG, Diouf $M$, Zogheib E, Dupont H. Learning curve for real-time ultrasoundguided percutaneous tracheostomy. Anaesth Crit Care Pain Med. 2017;36(5):279-83. https://doi.org/10.1016/j.accpm.2016.07.005 